\title{
Authentication Protocol for Healthcare Services over Wireless Body Area Networks
}

\author{
Seulgi Shin ${ }^{1}$, Sung Woon Lee ${ }^{2 *}$, Hyunsung Kim ${ }^{1}$ \\ 1 The Department of Cyber Security Kyungil University, Korea. \\ 2 The Department of Information Security, Tongmyong University, Korea. \\ * Corresponding author. Tel.: +82 10-6340-0587; email: staroun@tu.ac.kr \\ Manuscript submitted October 2, 2014; accepted April 25, 2015. \\ doi: 10.17706/ijcce.2016.5.1.50-60
}

\begin{abstract}
Ubiquitous healthcare service is one of the major fields of research for wireless body area networks (WBANs). Ensuring complete and a good level of security for such types of WBANs, is not a trivial task. It is practically impossible to deal with all sorts of security threats with a single mechanism. This paper reviews Khan et al.'s authentication protocol for healthcare service over WBANs and shows that it does not provide forward secrecy. Furthermore, this paper proposes a remedy protocol for Khan et al.'s authentication protocol and the previous related protocols. The proposed protocol could be utilized as a basic security building block for healthcare applications based on WBANs.
\end{abstract}

Key words: Healthcare service, wireless body area network, security, authentication.

\section{Introduction}

Wireless sensor networks (WSNs) are an emerging technology in existing research and have the potential to make human life more comfortable. Sensor node is the smallest unit of a network that has unique features like large scale deployment, mobility, reliability, and so on. A WSN consists of a group of nodes with low cost, low power, less memory, and limited computational power that communicate wirelessly over limited frequencies at low bandwidth [1], [2]. The main goals of WSNs are to deploy a number of sensor nodes over an unattended area, and collect the environmental data and transmit it to the base station or remote location. Later, the raw data is processed online or offline for detailed analysis at the remote server according to the application requirements.

At the same time, meeting the potential of WSNs in healthcare, called as wireless body area networks (WBANs) requires addressing a multitude of technical challenges. These challenges reach above and beyond the resource limitations that all WBANs face. Specifically, unlike applications in other domains, healthcare applications impose stringent requirements on system reliability, quality of service and particularly privacy and security [3]-[6].

Ensuring complete and a good level of security for such types of WBANs, is not a trivial task. As these types of networks use wireless communications, the threats and attacks against them are more diverse and often very large in scale. It is practically impossible to deal with all sorts of security threats with a single mechanism. Instead, a combination of different security schemes for a single network could be the solution [7]. After the development of simple user authentication schemes in [8]-[12], schemes for WSNs and WBANs have also attracted to the researchers. Some works has proposed for healthcare applications using WBANs [13]-[17]. Kumar et al. observed that most of the schemes in [8]-[10] fall short to provide security 
and also require heavy computational overhead and high communication cost and proposed a user authentication scheme named E-SAP [17]. Recently, Khan et al. showed that Kumar et al.'s scheme is weak against user impersonation attack, password guessing attack and node impersonation attack and lacks user anonymity. Furthermore, they proposed an improved user authentication protocol to solve the problems in Kumar et al.'s scheme [18].

This paper proposes a new user authentication protocol for healthcare services over WBANs. First of all, we will review Khan et al.'s protocol and show that it does not provide forward secrecy. After that, we will propose a new authentication protocol to solve the problem in Khan et al.'s protocol and the previous authentication protocols. The proposed protocol could provide anonymity and untraceability, which are very necessary properties in ubiquitous healthcare applications.

\section{Review of Khan et al.'s Authentication Scheme}

This section reviews network configuration of healthcare service based on WMSN, which is the focusing network environment in this paper. After that, we will review Khan et al.'s authentication scheme in [18] and show the security weakness in it.

\subsection{Network Configuration of Healthcare Service Based on WMSN}

There are four main parties in the WMSN, which are users, $M S$ nodes, $G W$ node and patients. Users are medical professionals like nurses, doctors, etc., looking for physiological data of the patient via WMSN. MS nodes are tiny sensors like temperature sensor, pulse oxi-meter, etc. deployed on the body of the patients. $G W$ node is a powerful master node which plays the role of the registering authority and acts as an interface between the user and the $M S$-node. Patients are under vigilance of medical professionals by means of $M S$ nodes for treatment (see Fig. 1).

$M S$ nodes are tiny sensors having low processing power, limited computational capabilities, and limited energy and storage capacity [19]. GW node is a powerful node with sufficiently large processing power, computational capabilities, and energy and storage capacity. A patient registers himself (or herself) to $G W$ node to become a valid participant of the system. Whenever a user wishes to obtain the physiological data of a patient, he (or she) transmits request message to $G W$ node of the patient. Afterwards, $G W$ node verifies the legitimacy of the user. Only if the verification is satisfied, directs the desired $M S$ node(s) to answer to the user's request.

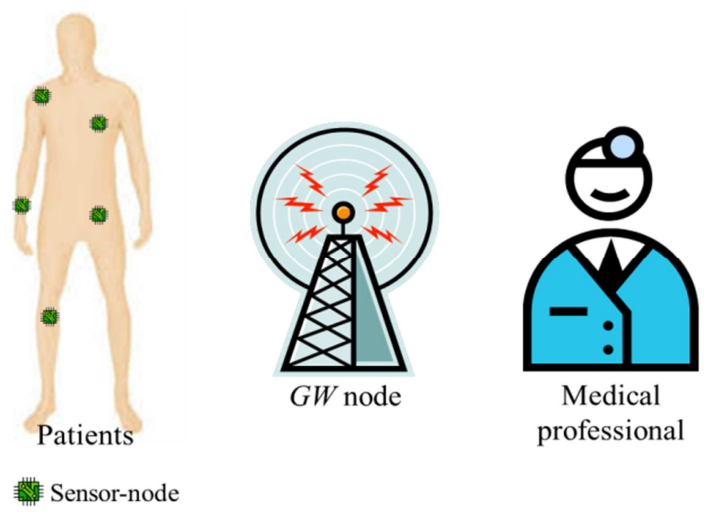

Fig. 1. Network configuration.

\subsection{Khan et al.'s Authentication Scheme}

This subsection reviews Khan et al.'s authentication scheme, which is consisted with five phases, user registration phase, patient registration phase, login phase, authentication phase and password change phase [18]. 
[User Registration Phase] The user (professional) $U$ registers itself to the $G W$-node in registration center of the hospital. In the following manner:

1) User choses her/his identity $I D_{u}$ and submits it to the $G W$ node using a secure channel.

2) On receiving $I D_{u}$ the $G W$ node computes $C_{u g}, K_{u}$, and $K_{g}$ as in (1), (2) and (3), respectively.

$$
\begin{gathered}
C_{u g}=E_{K}\left(I D_{u} \| I D_{g}\right) \\
K_{u}=h\left(K\left\|I D_{u}\right\| I D_{g}\right) \\
K_{g}=h\left(I D_{g} \| K\right)
\end{gathered}
$$

3) $G W$ node stores $\left\{h(),. C_{u g}\right\}$ into a $S C$, and provides $S C$ along with values $\left\{K_{u}, K_{g}\right\}$ to $U$ through the secure channel.

4) On obtaining $S C$ with the information $\left\{h(),. C_{u g}\right\}$ and $\left\{K_{u}, K_{g}\right\}$, the user $U$ chooses his (or her) password $P W_{u}$ and computes $N_{u}, P K_{u}$ and $P K_{g}$ as in (4), (5) and (6), respectively. Finally, $U$ inserts $N_{u}, P K_{u}$ and $P K_{g}$ in $S C$, so that $S C$ stores $\left\{h(),. C_{u g}, N_{u}, P K_{u}, P K_{g}\right\}$.

$$
\begin{aligned}
& N_{u}=h\left(I D_{u}\left\|P W_{u}\right\| K_{u}\right) \\
& P K_{u}=K_{u} \oplus\left(I D_{u} \| P W_{u}\right) \\
& P K_{g}=K_{g} \oplus\left(P W_{u} \| I D_{u}\right)
\end{aligned}
$$

[Patient Registration Phase] A patient has to register itself in registration center of the hospital. Patient submits his (or her) name to the registration center. On receiving patient's name, the registration center chooses a suitable medical sensor kit (i.e., $M S$ nodes and $G W$ node) according to the disease of the patient and assigns medical professionals (users). Then the registration center transmits the identity $I D_{p t}$ of the patient along with medical sensors kit information to the assigned professionals/users. Finally, a technician deploys $M S$-node on the body of the patient.

[Login Phase] A professional logs into the $G W$ node in order to gain patients' medical data via WMSN. The user inserts her/his $S C$ into the smart card reader and inputs $I D_{u}$ and $P W_{u}$. Then the $S C$ performs the following:

1) Retrieves $K_{u}$ and $K_{g}$ as in (7) and (8), and computes $N_{u}{ }^{*}$ as in (9). If $N_{u}{ }^{*}$ is the same with $N_{u}$, it continues further; otherwise stops the session.

$$
\begin{gathered}
K_{u}=P K_{u} \oplus\left(I D_{u} \| P W_{u}\right) \\
K_{g}=P K_{g} \oplus\left(P W_{u} \| I D_{u}\right) \\
N_{u}^{*}=h\left(I D_{u}\left\|P W_{u}\right\| K_{u}\right)
\end{gathered}
$$

2) Generates a random nonce $M$ and computes $C_{u 1}$ and $C I D_{u}$ as in (10) and (11), respectively.

$$
C_{u 1}=C_{u g} \oplus h\left(K_{g}\right)
$$




$$
C I D_{u}=E_{K_{u}}\left(h\left(I D_{u}\right)\|M\| S_{n}\left\|C_{u g}\right\| T_{u}\right)
$$

3) $S C$ sends $\left\{C I D_{u}, C_{u 1}, T_{u}\right\}$ as login request to the $G W$ node, where, $T_{u}$ is the current timestamp.

[Authentication Phase] When the login request $\left\{C I D_{u}, C_{u 1}, T_{u}\right\}$ from $U$ is received by the $G W$ node, it executes the following steps

1) Acquires current timestamp $T_{g}^{\prime}$ and if $\left(T_{g}^{\prime}-T_{u}\right) \leq \Delta T$. It discards the login request; otherwise proceeds further.

2) Retrieves $C_{u g}$ as in (12) and decrypts $C I D_{u}$ with $K_{u}$ to obtain $\left\{h\left(I D_{u}\right)^{\prime}, M, S_{n}, C_{u g}{ }^{*}\right.$ and $\left.T_{u}{ }^{*}\right\}$. Verifies whether $C_{u g}{ }^{*}$ is equivalent with $C_{u g}$. If it is correct, then decrypts $C_{u g}$ with $K$ to obtain $I D_{u}{ }^{*}$ and $I D_{g}$.

$$
C_{u g}=C_{u 1} \oplus h\left(K_{g}\right)
$$

3) Then computes $h\left(I D_{u}\right)^{*}$ and verifies whether $h\left(I D_{u}\right)^{*}, I D_{g}{ }^{*}$ and $T_{u}$ are equivalent with $h\left(I D_{u}\right)^{\prime}, I D_{g}$ and $T_{u}{ }^{*}$. If all the three equivalences hold, it believes that the login request is come from $U$; otherwise it terminates the session.

4) Acquires $T_{g}$ as current timestamp, computes $C_{g 1}$ as in (13) and sends $\left\{C_{g 1}, T_{g}\right\}$ to $U$. Acquires $T_{g s}$ as another current timestamp and computes $C_{g 2}$ and $A_{u}$ as in (14) and (15), respectively. Then, the $G W$ node sends $\left\{C_{g 2}, A_{u}, T_{g s}\right\}$ to the $M S$ node.

$$
\begin{gathered}
C_{g 1}=K_{g} \oplus\left(M\left\|T_{g}\right\| T_{u}\right) \\
C_{g 2}=h\left(K_{g s}\right) \oplus\left(C I D_{u}\left\|T_{g}\right\| M \| T_{u}\right) \\
A_{u}=h\left(C I D_{u}\left\|K_{g s}\right\| T_{g}\left\|S_{n}\right\| T_{g s}\right)
\end{gathered}
$$

On receiving $\left\{C_{g 1}, T_{g}\right\}$ from the $G W$ node, $U$ verifies the legitimacy of the $G W$ node as follows:

5) Checks if $\left(T_{u}^{\prime}-T_{g}\right) \leq \Delta T$. If so, dumps the session; otherwise continues further.

6) Obtains $\left(M^{*}\left\|T_{g}{ }^{*}\right\| T_{u}{ }^{*}\right)$ as in (16) and verifies whether $M^{*}$ and $T_{g}{ }^{*}$ are equivalent with $M$ and $T_{u}$. If they hold, then $G W$ node is authenticated; otherwise terminates the login session.

$$
\left(M^{*}\left\|T_{g}^{*}\right\| T_{u}^{*}\right)=C_{g 1} \oplus K_{g}
$$

After this mutual authentication, $U$ and $G W$ node compute Ksess $U$-GW as in (17) for the session key.

$$
\operatorname{Ksess}_{U-G W}=h\left(M\left\|I D_{u}\right\| T_{g}\right)
$$

On receiving $\left\{C_{g 2}, A_{u}, T_{g s}\right\}$ from the $G W$ node, the $M S$ node performs the following operations:

7) Checks if $\left(T_{s}^{\prime}-T_{g s}\right) \leq \Delta T$. If so, terminates the session; otherwise proceeds further.

8) Obtains $\left(C I D_{u}{ }^{*}\left\|T_{g}{ }^{*}\right\| M^{*} \| T_{u}{ }^{*}\right)$ as in (18), computes $A_{u}{ }^{*}$ as in (19) and compares $A_{u}{ }^{*}$ with $A_{u}$. If the verification holds, the legitimacy of the $G W$ node is successful and hence of $U$.

$$
\begin{gathered}
\left(C I D_{u}^{*}\left\|T_{g}^{*}\right\| M^{*} \| T_{u}^{*}\right)=C_{g 2} \oplus h\left(K_{g s}\right) \\
A_{u}^{*}=h\left(C I D_{u}^{*}\left\|K_{g s}\right\| T_{g}^{*}\left\|S_{n}\right\| T_{g s}\right)
\end{gathered}
$$


9) Acquires $T_{s g}$ as current timestamp, computes $C_{s 1}$ as in (20), and sends $\left\{C_{s 1}, T_{s g}\right\}$ to the $G W$ node. Also computes $C_{s 2}{ }^{*}$ as in (21), where $T_{s}$ is another current timestamp of $M S$ node. Then, the $M S$ node sends $\left\{C_{s 2}{ }^{*}, T_{s}\right\}$ to $U$.

$$
\begin{gathered}
C_{s 1}=h\left(T_{g}\left\|K_{g s}\right\| T_{s g}\right) \oplus h\left(C I D_{u} \| S_{n}\right) \\
C_{s 2}^{*}=h\left(S_{n}\left\|M^{*}\right\| T_{u} \| T_{s}\right)
\end{gathered}
$$

On receiving $\left\{C_{s 1}, T_{s g}\right\}$ from the $M S$ node, the $G W$ node performs the following operations:

10) Checks if $\left(T_{g}{ }^{\prime \prime}-T_{s g}\right) \leq \Delta T$, if so, terminates the session; otherwise proceeds further.

11) Obtains $\left(h\left(C I D_{u}|| S_{n}\right)\right)^{*}$ as in (22), and compares it with $h\left(C I D_{u} \| S_{n}\right)$. The equivalence whether $\left(h\left(C I D_{u} \| S_{n}\right)\right)^{*}$ is equivalent with $h\left(C I D_{u} \| S_{n}\right)$ verifies the legitimacy of $M S$ node.

$$
\left(\mathrm{h}\left(C I D_{u} \| S_{n}\right)\right)^{*}=C_{s 1} \oplus h\left(T_{g}\left\|K_{g s}\right\| T_{s g}\right)
$$

After this mutual authentication, $G W$ node and $M S$ node compute $K \operatorname{sess}_{G W-S n}$ as in (23) for the session key.

$$
\operatorname{Ksess}_{G W-S_{n}}=h\left(K_{g s}\left\|T_{s g}\right\| M\right)
$$

On receiving $\left\{C_{s 2^{*}}{ }^{*}, T_{s}\right\}$ from the $M S$ node, $U$ performs the following:

12) Checks if $\left(T_{u}{ }^{\prime \prime}-T_{s}\right) \leq \Delta T$. If so, dumps the session, otherwise proceeds further.

13) Computes $C_{s 2}$ as in (24) and compares it with $C_{s 2}{ }^{*}$. If $C_{s 2}$ is equal to $C_{s 2}{ }^{*}$, the authenticity of $M S$ node is verified.

$$
C_{s 2}=h\left(S_{n}\|M\| T_{u} \| T_{s}\right)
$$

After this mutual authentication, $U$ and $M S$ node compute Ksess $U$-Sn as in (25) for the session key.

$$
\operatorname{Ksess}_{U-S_{n}}=h\left(M\left\|T_{S}\right\| S_{n}\right)
$$

[Password Change Phase] $U$ can change his (or her) password in the following manner. For this, $U$ inserts his (or her) $S C$ into the terminal, inputs his (or her) $I D_{u}$ and $P W_{u}$, and opts to change his (or her) password. Then the following steps are performed to update a new password:

1) $S C$ retrieves $K_{u}$ and $K_{g}$ as in (7) and (8), and computes $N_{u}{ }^{*}$ as in (9). If $N_{u}{ }^{*}$ is equivalent to $N_{u}$, then proceeds further after asking for new password; otherwise discards the password change request.

2) $U$ enters a new password $\left(P W_{u}\right)_{\text {new. }}$.

3) SC computes with $\left(N_{u}\right)_{n e w},\left(P K_{u}\right)_{\text {new }}$ and $\left(P K_{g}\right)_{\text {new }}$ as in (26), (27) and (28), respectively.

$$
\begin{aligned}
& \left(N_{u}\right)_{\text {new }}=h\left(I D_{u}\left\|\left(P W_{u}\right)_{\text {new }}\right\| K_{u}\right) \\
& \left(P K_{u}\right)_{\text {new }}=K_{u} \oplus\left(I D_{u} \|\left(P W_{u}\right)_{\text {new }}\right) \\
& \left(P K_{g}\right)_{\text {new }}=K_{g} \oplus\left(\left(P W_{u}\right)_{\text {new }} \| I D_{u}\right)
\end{aligned}
$$

4) $S C$ replaces $N_{u}, P K_{u}$ and $P K_{g}$ with $\left(N_{u}\right)_{\text {new }},\left(P K_{u}\right)_{\text {new }}$ and $\left(P K_{g}\right)_{\text {new, }}$, respectively. 


\subsection{Security Analysis on Khan et al.'s Scheme}

Khan et al. argued that their scheme is secure against various attacks and provides good properties. However, this section shows that Khan et al's scheme does not provide forward secrecy, which is necessary property to be supported to the key agreement scheme. We need to have an assumption that attacker could get the system's long term secret key $K$ as usual for the forward secrecy. Also, we need another assumption that attacker also could steal and read the smart card.

For the attack, first of all, an attacker could derive $I D_{u}{ }^{\prime}$ and $I D_{g}{ }^{\prime}$ by decrypting $C_{u g}$ on the smart card. After that, the attacker could derive $K_{g}{ }^{\prime}$ with the long term secret key $K$ and the identity of GW node $I D_{g}{ }^{\prime}$. Note that $K_{u}$ works as a very important key for the confidentiality of communication in between. The attacker could know $K_{u}{ }^{\prime}$ from the derivations by computing $h\left(K|| I D_{u}{ }^{\prime}|| I D_{g}{ }^{\prime}\right)$ and $K_{g s}{ }^{\prime}$ as in (29) and decrypt $C I D_{u}$ by using $K_{u}$ to derive $M^{\prime}$ and $S_{n}{ }^{\prime}$. Then, the attacker could derive two session keys Ksess $_{U-G W^{\prime}}{ }^{\prime}$ and Ksess $_{U-S n^{\prime}}{ }^{\prime}$ as in (30) and (31) properly. Thereby, Khan et al's scheme does not provide forward secrecy.

$$
\begin{gathered}
K_{g s}{ }^{\prime}=h\left(K \| I D_{g}{ }^{\prime}\right) \\
\operatorname{Ksess}_{U-G W}{ }^{\prime}=h\left(M^{\prime}\left\|I D_{u}{ }^{\prime}\right\| T_{g}\right) \\
\operatorname{Ksess}_{U-S_{n}}=h\left(M^{\prime}\left\|T_{s}\right\| S_{n}{ }^{\prime}\right)
\end{gathered}
$$

\section{Proposed Authentication Scheme}

This section proposes a new user authentication protocol for healthcare services over WBANs. The proposed protocol could provide anonymity and untraceability by adopting dynamic identity depending on the session fresh random numbers. The proposed protocol is also consisted with five phases including user registration phase, patient registration phase, login phase, authentication phase and password change phase.

\subsection{User Registration Phase}

The user (medical professional) $U$ registers himself (or herself) to the $G W$ node in registration center of the hospital in the following manner:

1) User chooses her/his identity $I D_{u}$ and submits it to the $G W$ node using a secure channel.

2) On receiving $I D_{u}$, the $G W$ node computes $C_{u g}, K_{u}$, and $K_{g}$ as in (1), (2) and (3), respectively.

3) $G W$ node stores $\left\{h(),. C_{u g}\right\}$ into a $S C$, and provides $S C$ along with values $\left\{K_{u}, K_{g}\right\}$ to $U$ through the secure channel.

4) On obtaining $S C$ with the information $\left\{h(),. C_{u g}\right\}$ and $\left\{K_{u}, K_{g}\right\}$, the user $U$ chooses his (or her) password $P W_{u}$ and computes $N_{u}, P K_{u}$ and $P K_{g}$ as in (4), (5) and (6), respectively. Finally, $U$ inserts $N_{u}, P K_{u}$ and $P K_{g}$ in $S C$, so that $S C=\left\{h(),. C_{u g}, N_{u}, P K_{u}, P K_{g}\right\}$.

\subsection{Patient Registration Phase}

A patient has to register himself (or herself) in registration center of the hospital. Patient submits his (or her) name to the registration center. On receiving the patient's name, the registration center chooses a suitable medical sensor kit (i.e., $M S$ nodes and $G W$ node) according to the disease of the patient and assigns medical professionals. Then, the registration center transmits the identity $I D_{p t}$ of the patient along with medical sensors kit information to the assigned professionals/users. Finally, a technician deploys the $M S$ node on the body of the patient. 


\subsection{Login Phase}

A professional logs into the $G W$ node in order to gain patients' medical data via a WMSN. The user inserts his (or her) $S C$ into the smart card reader and inputs $I D_{u}$ and $P W_{u}$. Then the $S C$ performs the following processes:

1) $S C$ retrieves $K_{u}$ as in (7), $K_{g}$ as in (8) and computes $N_{u}{ }^{*}$ as in (9). If $N_{u}{ }^{*}$ is equal to $N_{u}$, $S C$ continues further; otherwise $S C$ stops the session.

2) Generates a random nonce $a$ and computes $A$ as in (32), $C_{u 1}$ as in (10) and $C I D_{u}$ as in (11).

$$
A=g^{a}
$$

3) SC sends $\left\{C I D_{u}, C_{u 1}\right\}$ as login request to $G W$ node.

\subsection{Authentication Phase}

When the login request $\left\{C I D_{u}, C_{u 1}\right\}$ from $U$ is received by the $G W$ node, it executes the following steps:

1) The $G W$ node retrieves $C_{u g}$ as in (12) and decrypts $C I D_{u}$ as $D_{K}\left(C I D_{u}\right)$ to obtain $\left\{h\left(I D_{u}\right)^{\prime}, A, S_{n}{ }^{*}\right.$ and $\left.C_{u g}{ }^{*}\right\}$. The $G W$ node verifies whether $C_{u g}{ }^{*}$ is equivalent with $C_{u g}$. If correct, then the $G W$ node decrypts $C_{u g}$ as $D_{K u}\left(C_{u g}\right)$ to obtain $I D_{u}{ }^{*}$ and $I D_{g}$.

2) Then the $G W$ node computes $h\left(I D_{u}\right)^{*}$ and verifies whether $h\left(I D_{u}\right)^{*}, I D_{g}{ }^{*}$ are equivalent with $h\left(I D_{u}\right)^{\prime}, I D_{g}$. If all equivalences hold, the $G W$ node believes the login request is come from $U$; otherwise the $G W$ node terminates the login session.

3) The $G W$ node generates a random nonce $b$ and computes $B$ as in (33), $S K_{G W-U}$ as in (34), $C_{g 1}$ as in (35) and $A_{m}$ as in (36) and sends $\left\{C_{g 1}, A_{m}\right\}$ to $U$. The $G W$ node computes, $C_{g 2}$ as in (37) and $A_{u}$ as in (38). Then, the $G W$ node sends $\left\{C_{g 2}, A_{u}\right\}$ to the $M S$ node.

$$
\begin{gathered}
B=g^{b} \\
S K_{G W-U}=A^{b} \\
C_{g 1}=K_{g} \oplus(A \| B) \\
A_{m}=h\left(S K_{U-G W}\|A\| B\right) \\
C_{g 2}=h\left(K_{g s}\right) \oplus\left(C I D_{u}\|A\| B\right) \\
A_{u}=h\left(K_{g s}\left\|S_{n}\right\| A \| B\right)
\end{gathered}
$$

On receiving $\left\{C_{g 1}, A_{m}\right\}$ from the $G W$ node, $U$ verifies the legitimacy of the $G W$ node as follows:

4) $U$ obtains $\left(A^{*} \| B^{*}\right)$ as in (39) and verifies $A_{m}$ and checks $A^{*}$ is equal with $A$. For $A_{m}$ verification, $U$ computes $S K_{U-G W}$ as in (40) and $A_{m}{ }^{*}$ as in (41) and checks $A_{m}{ }^{*}$ is equal with $A_{m}$. Only if they hold, the $G W$ node is authenticated and both of $U$ and the $G W$ node share a session key $S K_{U-G W}$, which is the same with $S K_{G W-U}$ correctly; otherwise $U$ terminates the login session.

$$
\begin{gathered}
\left(A^{*} \| B^{*}\right)=C_{g 1} \oplus K_{g} \\
S K_{U-G W}=B^{a}
\end{gathered}
$$




$$
A_{m}^{*}=h\left(S K_{U-G W}\|A\| B^{*}\right)
$$

On receiving $\left\{C_{g 2}, A_{u}\right\}$ from the $G W$ node, the $M S$ node performs the following operations

5) The $M S$ node obtains $\left(C I D_{u}{ }^{*} \| A^{*}|| B^{*}\right)$ as in (42), computes $A_{u}{ }^{*}$ as in (43) and compares $A_{u}{ }^{*}$ with $A_{u}$. The equivalence check between $A_{u}{ }^{*}$ and $A_{u}$ verifies the legitimacy of the $G W$ node and hence of $U$.

$$
\begin{gathered}
\left(C I D_{u}^{*}\left\|A^{*}\right\| B^{*}\right)=C_{g 2} \oplus h\left(K_{g s}\right) \\
A_{u}^{*}=h\left(K_{g s}\left\|S_{n}\right\| A^{*} \| B^{*}\right)
\end{gathered}
$$

6) The $M S$ node generates a random nonce $f$ and computes $F$ as in (44), $S K_{M S-G W}$ as in (45), $C_{s 1}$ as in (46) and $A_{m s}$ as in (47) and sends $\left\{C_{s 1}, A_{m s}\right\}$ to the $G W$ node. Also the $M S$ node computes $S K_{M S-U}$ as in (48), $C_{s 2}$ as in (49) and $A_{m s u}$ as in (50). Then, the $M S$ node sends $\left\{C_{s 2}, A_{m s u}\right\}$ to $U$.

$$
\begin{gathered}
F=g^{f} \\
S K_{M S-G W}=B^{* f} \\
C_{s 1}=h\left(C I D_{u} \| S_{n}\right) \oplus F \\
A_{m s}=h\left(S K_{M S-G W}\left\|K_{g s}\right\| B^{*} \| F\right) \\
S K_{M S-U}=A^{* f} \\
C_{s 2}=A^{*} \oplus F \\
A_{m s u}=h\left(S K_{M S-U}\left\|S_{n}\right\| A^{*} \| F\right)
\end{gathered}
$$

On receiving $\left\{C_{s 1}, A_{m s}\right\}$ from the $M S$ node, the $G W$ node performs the following operations:

7) The $G W$ node obtains $F^{*}$ as in (51), $S K_{G W-M S}$ as in (52) and $A_{m s}{ }^{*}$ as in (53). The equivalence check between $A_{m s}{ }^{*}$ and $A_{m s}$ verifies the legitimacy of the $M S$ node and the session key agreement.

$$
\begin{gathered}
F^{*}=C_{s 1} \oplus h\left(C I D_{u} \| S_{n}^{*}\right) \\
S K_{G W-M S}=F^{* b} \\
A_{m s}^{*}=h\left(S K_{G W-M S}\left\|K_{g s}\right\| B^{*} \| F\right)
\end{gathered}
$$

On receiving $\left\{C_{s 2}, A_{m s u}\right\}$ from the $M S$ node, $U$ performs the following steps

8) $U$ computes $F^{*}$ as in (54), $S K_{U-M S}$ as in (55) and $A_{m s u}{ }^{*}$ as in (56) and compares $A_{m s u}{ }^{*}$ with $A_{m s u}$. Only if they match, $U$ verifies the legitimacy of the $M S$ node and the session key agreement.

$$
\begin{gathered}
F^{*}=C_{S 2} \oplus A \\
S K_{U-M S}=F^{*}
\end{gathered}
$$




$$
A_{m s u}^{*}=h\left(S K_{U-M S}\left\|S_{n}\right\| A \| F^{*}\right)
$$

\subsection{Password Change Phase}

$U$ can change his (or her) password in the following manner. For this, $U$ inserts his (or her) $S C$ into the terminal, inputs his (or her) $I D_{u}$ and $P W_{u}$, and opts to change his (or her) password. Then the following steps are performed to update a new password

1) $S C$ retrieves $K_{u}$ as in (7), $K_{g}$ as in (8) and computes $N_{u}{ }^{*}$ as in (9). If $N_{u}{ }^{*}$ is equivalent to $N_{u}$, then proceeds further after asking for new password; otherwise discards the password change request.

2) $U$ enters a new password $\left(P W_{u}\right)_{\text {new. }} S C$ computes $\left(N_{u}\right)_{\text {new }}$ as in (26), $\left(P K_{u}\right)_{\text {new }}$ as in (27) and $\left(P K_{g}\right)_{\text {new }}$ as in (28). $S C$ replaces $N_{u}, P K_{u}$ and $P K_{g}$ with $\left(N_{u}\right)_{\text {new, }}\left(P K_{u}\right)_{\text {new }}$ and $\left(P K_{g}\right)_{\text {new, }}$, respectively.

\section{Security Analyses}

This section provides security analysis of the proposed protocol. We will consider that the proposed protocol is secure under the same assumption subject to which Khan et al.'s protocol is attackable. The assumption is that an attacker $U_{a}$ can extract the information stored inside the smart card. Also, we need to have an assumption that attacker could get the system's long term secret key $K$ as usual for the forward secrecy.

\subsection{Provides User Anonymity}

If $U_{a}$ intercepts the login request $\left\{C I D_{u}, C_{u 1}\right\}$ of $U, U_{a}$ needs $K_{u}$ to obtain $h\left(I D_{u}\right)$ by decrypting $C I D_{u}$. But $U_{a}$ neither knows $K_{u}$ nor recovers $h\left(I D_{u}\right)$ by extracting information $\left\{h(),. C_{u g}, N_{u}, P K_{u}, P K_{g}\right\}$ from the lost smart card of $U$. To take out $K_{u}$ from $P K_{u}, U_{a}$ should know user's identity and password. In fact, $K_{u}$ required to encrypt or decrypt $C I D_{u}$ is not stored directly in user's smart card and is different for each user. Therefore, $U_{a}$ cannot obtain $h\left(I D_{u}\right)$. On the other hand, to procure identity $I D_{u}$ from $N_{u}, P K_{\underline{u}}$ or $P K_{g}$ is infeasible. It requires knowledge of keys $K_{u}$ and $K_{g}$ to gain $I D_{u}$ out of $P K_{u}$ or $P K_{g}$ respectively. Moreover, one-way property of hash function does not allow the extraction of $I D_{u}$ out of $N_{u}$. Therefore, $U_{a}$ cannot gain the identity of a user and hence the protocol provides user anonymity.

\subsection{Resists Password Guessing Attack}

In order to guess $U^{\prime}$ s password $P W_{u}$ obtained from the lost $S C$ of $U, U_{a}$ requires knowledge of $I D_{u}$ and $K_{u}$. As described in the user anonymity part, $U_{a}$ cannot gain the identity of a user either from the lost smart card of a user or from an intercepted login request. Besides, $K_{u}$ is not available as plaintext in $U$ 's $S C$ and is not obtainable from $P K_{u}$ without having exact values of $I D_{u}$ and $P W_{u}$. Thus, the protocol resists password guessing attack.

\subsection{Provides Secure Session Key between Every Entities}

The proposed protocol establishes session key between every pair of participants. Session key between $U$ and the $G W$ node is computed as in (34), which depends on session dependent random numbers from each entity. $U_{a}$ cannot compute $S K_{U-G W}$ even if the case that $U_{a}$ knows $A$ and $B$ due to the discrete logarithm problem. Session key between the GW node and the $M S$ node is computed as in (52) which is dynamic because of session dependent random number $b$ and $f$. Session key between $U$ and the $M S$ node is computed as in (55) which $U_{a}$ cannot compute without knowing $a$ and $f$. Thus, the protocol establishes independent and secure session keys between every pair of the participants.

\subsection{Provides Forward Secrecy}

If we have two assumptions as the attack to Khan et al.'s scheme, the proposed protocol provides forward 
secrecy. $U_{a}$ should be able to get the messages $\left\{C I D_{u}, C_{u 1}\right\},\left\{C_{g 2}, A_{u}\right\},\left\{C_{s 1}, A_{m s}\right\}$ and $\left\{C_{s 2}, A_{m s u}\right\}$. However, there is no way $U_{a}$ to know about $S K_{U-G W}, S K_{G W-M S}$ and $S K_{U-M S}$ due to the discrete logarithm problem. Thereby, the proposed protocol provides forward secrecy.

\section{Conclusion}

This paper has been proposed a new user authentication protocol for healthcare services over WBANs. First of all, we reviewed Khan et al's protocol and showed that it does not provide forward secrecy. After that, we proposed a new authentication protocol to solve the problem in Khan et al's protocol and the previous authentication protocols. The proposed protocol could provide anonymity and untraceability, which are very necessary properties in ubiquitous healthcare applications.

\section{Acknowledgment}

This work was supported by the National Research Foundation of Korea Grant funded by the Korean Government (MEST) (NRF-2011-0008890) and also was supported by the National Research Foundation of Korea Grant funded by the Korean Government (MEST) (NRF-2010-0021575).

\section{References}

[1] Ko, B. J. G., Lu, C., Srivastva, M. B., Stankovic, J., Terzis, A. A., \& Welsh, M. (2010). Wireless sensor network for healthcare. Proceedings of IEEE, 98(11), 1947-1960.

[2] Kumar, P., \& Lee, H. (2012). Security issues in healthcare applications using wireless medical sensor networks: A survey. Sensors. 12(1), 55-91.

[3] Caytiles, R. D., \& Park, S. (2014). A study of the design of wireless medical sensor network based u-Healthcare system. International Journal of Bio-Science and Bio-Technology, 6(3), 91-96.

[4] Kim, H., Ryu, E. K., \& Lee, S. W. (2013). Security considerations on cognitive radio based on body area networks for u-Healthcare. Journal of Security Engineering, 10(1), 9-20.

[5] Kim, H., \& Lee, S. W. (2009). Enhanced novel access control protocol over wireless sensor networks. IEEE Trans. on Consumer Electronics, 55(2), 492-498.

[6] Mtonga, K., Yoon, E. J., \& Kim, H. (2014). A pairing based authentication and key establishment scheme for remote patient monitoring systems. Lecture Notes of the Institute for Computer Sciences, 135, 79-89.

[7] Haque, M., Pathan, A. K., \& Hong, C. S. (2008). Securing U-healthcare sensor networks using public key based scheme. Proceedings of $10^{\text {th }}$ International Conference on Advanced Communication Technology: Vol. 2 (pp. 1108-1111).

[8] Kim, H., Lee, S. W., \& Yoo, K. Y. (2003). ID-based password authentication scheme using smart cards and fingerprints. Operating Systems Review (pp. 32-41).

[9] Lee, S. W., Kim, H., \& Yoo, K. Y. (2004). Improved efficient remote user authentication scheme using smartcards. IEEE Trans. on Consumer Electronics, 50(2), 565-567.

[10] Lee, S. W., Kim, H., \& Yoo, K. Y. (2005). Improvement of Chien et al's remote user authentication scheme using smart cards. Computer Standards and Interfaces, 27, 181-183.

[11] Kim, H. (2011). Location-based authentication protocol for first cognitive radio networking standard. Journal of Network and Computer Applications, 34, 1160-1167.

[12] Vallent, T. F., \& Kim, H. (2013). Three factor authentication protocol based on bilinear pairing. Lecture Notes in Electrical Engineering, 240, 253-260.

[13] Saleem, S., Ullah, S., \& Yoo, H. S. (2009). On the security issues in wireless body area networks. Journal of Digital Content Technology and Its Applications, 3(3), 178-184.

[14] Ullah, S., \& Kwak, K. (2011). Body area network for ubiquitous healthcare applications: Theory and 
implementation. Journal of Medical Systems, 35(5), 1243-1244.

[15] Ullah, S., Higgins, H., Braem, B., Latre, B., Blondia, C., Moerman, I., Saleem, S., Rahman, Z., \& Kwak, K. (2012). A comprehensive survey of wireless body area networks - on PHY, MAC, and network layers solutions. Journal of Medical Systems, 36(3), 1065-1094.

[16] Chung, W. Y. (2012). Multi-modal sensing M2M healthcare service in WSN. KSII Transactions on Internet and Information Systems, 6(4), 1065-1094.

[17] Kumar, P., Lee, S. G., \& Lee, H. J. (2012). E-SAP: Efficient-strong authentication protocol for healthcare applications using wireless medical sensor networks. Sensors, 12, 1625-1647.

[18] Khan, M. K., \& Kumari, S. (2014). An improvement user authentication protocol for healthcare services via wireless medical sensor networks. International Journal of Distributed Sensor Networks.

[19] Mtonga, K. (2013). Secure Authentication Scheme for Remote Health Monitoring System Using WBAN. M. S. Thesis, Kyungil University.

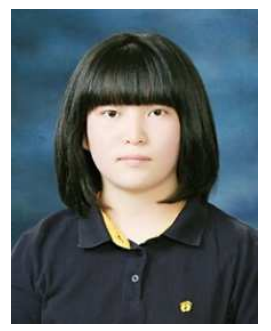

Seulgi Shin is a student at the Department of Cyber Security Kyungil University, Republic of Korea from 2013. She is interested in information security protocol, wireless sensor network security, ubiquitous computing security, cloud computing security, medical healthcare security, and recent information security issues.

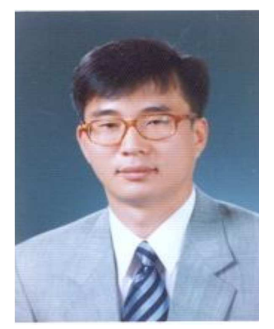

Sung Woon Lee is a professor at the Department of Information Security, Tongmyong University, Korea. He received the B.S. and M.S. degrees in computer science from Chonnam National University, Korea in 1994 and 1996, respectively, and the Ph.D. degree in computer engineering from Kyungpook National University, Korea, in 2005. He was with the Korea Information System as a researcher, Korea, from 1996 to 2000. His research interests include cryptography, network security, and security protocol.

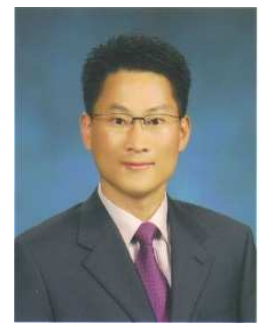

Hyunsung Kim is a professor at the Department of Cyber Security, Kyungil University, Korea. He received his M.S. and Ph.D. degrees in computer engineering from Kyungpook National University, Republic of Korea, in 1998 and 2002, respectively. From 2000 to 2002, he worked as a senior researcher at Ditto Technology. He had been an associate professor from 2002 to 2011 with the Department of Computer Engineering, Kyungil University. He had been a visiting scholar from 2009 to 2010 with the School of Computing, Dublin City University, Ireland. Currently, he is a full professor at the Department of Cyber Security, Kyungil University. His current research interests are cryptography, VLSI, authentication technologies, network security and ubiquitous computing security. 